\title{
Apps and More: Potentials of Digitalization in Breast Medicine
}

\author{
Friedrich Overkamp \\ onkowissen.de GmbH, Würzburg, Germany
}

Keywords

Digitalization · Apps · Breast cancer

\section{Abstract}

Digitalization in medicine is already being welcomed by some as a revolution and a deliverance, while others are still tending to take a more critical view of it. Digital tools are certainly still unfamiliar to the majority of physicians, and their potential benefits may not be immediately clear. As digitalization continues to advance, however, several exciting developments are emerging that may also be of interest in gynecology, and in particular for gynecological oncology and breast medicine. This note aims to outline a few examples in three areas: wearables, telemedicine, and "big data." Two other areas of development - digital follow-up and digital knowledge transfer - are also discussed in more detail.

C 2019 S. Karger AG, Basel

\section{Introduction}

Digitalization in medicine is already being welcomed by some as a revolution and a deliverance, while others are still tending to take a more critical view of it. Digital tools are certainly still unfamiliar to the majority of physicians, and their potential benefits may not be immediately clear.

As digitalization continues to advance, however, several exciting developments are emerging that may also be of interest in gynecology, and in particular for gynecological oncology and breast medicine. This note aims to outline a few examples in three areas: wearables, telemed- icine, and "big data." Two other areas of development digital follow-up and digital knowledge transfer - are also discussed in more detail.

\section{Wireless Sensors}

"Wearables" are devices with wireless sensors that are able to measure various parameters and send the results immediately to a mobile terminal device such as a smartphone or smart watch. This has already been implemented for measurements of blood sugar, blood pressure, pulse rate, and electrocardiography. AliveCor ${ }^{\circledR}$ and Apple Watch $4^{\circledR}$ are examples of wireless electrocardiography data transmission that have already received approval from the authorities in the United States. A smart pill containing the antipsychotic agent aripiprazole (Abilify Mycite $^{\circledR}$ ) is already on the market in the USA. An ingestible event marker integrated in a tablet sends information to the patient's smartphone when it comes into contact with gastric acid - a prototype for digital adherence checking.

\section{Telemedicine}

Treatment at a distance (telemedicine) may be particularly helpful for patients who live in rural areas. Even independently of geographical location, however, there are many potential areas in which telemedicine could be useful in breast medicine. One example might be followup of the cutaneous side effects of targeted or immunooncological cancer treatment, which in many cases can be

\section{KARGER}

(C) 2019 S. Karger AG, Basel 
done using a photo taken on a smartphone just as well as with a personal consultation. Video conferences with leading experts to discuss complex cases, or for molecular tumor board discussions, are likely to become everyday reality in oncological care in the near future. In the field of robotic surgery, intraoperative telemedicine consultations are already being used today for special issues arising in gyneco-oncological procedures.

\section{"Big Data"}

"Big data" will have a particularly strong influence on diagnosis and treatment in the field of oncology. Algorithm-based, learning-capable programs in radiology and pathology are opening up fresh opportunities in the diagnostic field - as well as creating new challenges. To optimize therapy, the sometimes gigantic collections of clinical and molecular-biological data that are being collected on breast carcinoma are likely to lead to increasing use of molecular-guided treatment for various subgroups of patients. Another fascinating development involves learning-capable programs based on artificial intelligence (e.g., $\left.\mathrm{Ada}^{\circledR}\right)$, which are providing suspected diagnoses on the basis of details from a patient's history, with increasingly high levels of accuracy.

\section{Digital Follow-Up}

Digital follow-up during cancer treatment is emerging as an important field for digitalization in oncology. Since the 2017 Annual Meeting of the American Society of Clinical Oncology (ASCO), it has been recognized that digital tools may be extremely valuable for follow-up purposes. The study by Basch et al. [1] clearly showed improved survival outcome for patients in a digital intervention arm compared to those with "classic" medical followup examinations. Patients being randomized to the intervention arm were asked to document their adverse events and a nurse actively contacted the patients with relevant adverse events. This was probably the reason for improved outcomes.

This scientific finding is already being put into practice in Germany using various tools, including the Cankado ${ }^{\circledR}$ program - an online diary for cancer patients that can be used to measure compliance, quality of life, and patientreported outcomes. The Cankado ${ }^{\circledR}$ program has been introduced in 2015 as an eHealth platform designed to support therapy management and interaction between patients and physicians and is already being used in clinical routine.

As a certified medical product, the program can also be used for treatment management; reminding patients to take their medication is the simplest way of increasing compliance during oral therapy [2]. The Cankado ${ }^{\circledR}$ app (https://cankado.com) makes it possible to issue dayvariable and period-specific reminders to take medication, specifically in oncology, and it is already being used in research studies with breast carcinoma patients.

Another major success factor for this type of device in cancer treatment is that it enables patients to understand why taking the medicine as prescribed is important for them, what symptoms may occur, and how they should deal with them. This has been investigated in the multicenter Patient Competence in Oral Cancer Therapy (PACOCT) study, in which Welslau et al. [3], in collaboration with the Scientific Institute of Private-Practice Hematologists and Oncologists (Wissenschaftliches Institut der Niedergelassenen Hämatologen und Onkologen, WINHO), showed that providing the patient with a nonmedical coach in oral cancer therapy improves processes, reduces side effects, and can thus also increase patients' willingness to take the prescribed medication [3]. With regard to these findings, medical personnel should be better integrated to support oncologic treatment.

The POSCA study, in which the patient coaching methods used in the PACOCT study are combined with the Cankado online platform, is developing these findings further. In this study, also conducted by Welslau et al., it has been shown that with coaching, even patients over the age of 80 are able to become active members of their own care team and are able to document the course of their disease on the computer [4].

Another application in usage for patient-reported outcomes and care management is PiiA, which has also been published previously.

The PiiA digital health companion is a web-based, patient-centered care planning and engagement tool that has been validated for quality of life assessments such as EORTC QLQ C30 and FACT-B [5, 6]. The application is implemented in the PRAEGNANT multicenter trial for patient-reported outcome data collection in metastatic breast cancer [7]. Furthermore, patient adoption, even within the age range of over 60 years, has been high [8].

In routine care, it has previously been customary to use patient identification cards and treatment diaries to document that medication has been taken. In the future, patients will increasingly exchange information in forums and blogs and will use digital tools to guide and document their treatment.

These examples illustrate the options that are emerging for digital technology in the fields of gynecological oncology and breast medicine. An indispensable prerequisite will of course be that the methods are subject to certification processes and are validated in research studies. 


\section{Digital Knowledge Transfer}

Another field in which digital technology is being increasingly widely used is that of digital knowledge transfer. With the development of smartphones and tablets, substantial amounts of Internet usage have been moving from classic websites to apps for some 10 years already. Various services are currently under development, including the apps developed by onkowissen.de. These apps process the current state of knowledge and options available in such a way that they can be made available effortlessly at any time on smartphones and tablets. Although the facilities have commercial support, they aim to be academic tools providing product-neutral information, reflecting the current state of knowledge with a modern app design and taking all the national and international guidelines into account.

The apps are prepared by an editorial team that includes scientists, pharmacists, and physicians, and they are reviewed and monitored by interdisciplinary committees including renowned and respected experts. The apps are available for download free of charge from the app stores and are also accessible as a classic website through onkowissen.de. Several apps on various different tumor entities are already available, including breast carcinoma. In the longer term, a digital compendium is planned that will cover the major areas of oncological knowledge and will be constantly updated.

The "onkowissen breast carcinoma" app (https://mammakarzinom.onkowissen.de) provides brief and concise descriptions of evidence-based diagnostic and therapeutic measures. "Mind maps" provide innovative guidance regarding possible sequential treatments. Pioneering original literature and the underlying guidelines are listed as sources for each topic, and the service is accompanied by a detailed reference list. The available drugs are included in the articles, and they are also retrievable via an alphabetic list. The specialist information can be called up when needed directly from the app. Wherever helpful, established tools such as follow-up schedules can be directly printed out from the app. The approved and established treatment approaches are included, and drug interactions, drug class-specific side effects and the appropriate supportive therapy are presented in detail. The "updates" heading will regularly draw attention to pioneering publications and use short videos to report on highlights from world conferences. These apps are designed for physicians and are therefore only usable with a password from DocCheck or onkowissen.de.

Another app that may be of interest to gynecologists and physicians in breast medicine is "onkowissen supportive therapy" (https://supportiv.onkowissen.de/), which covers most important areas of side effect management. In view of the special importance of osteo-oncolo- gy, the supportive therapy app is supplemented with an "onkowissen osteooncology" app (https://osteoonkologie.de/) dealing with specific issues. An "onkowissen biosimilars" app (https://biosimilars.onkowissen.de/) is also available. It provides information about production processes, approval, and safety aspects of biosimilar antibodies, and about the worldwide status of biosimilar development.

The onkowissen.tv video platform (https://www. onkowissen.tv/) will be supplementing the apps by reporting regularly on important world conferences - e.g., ASCO, the European Society for Medical Oncology (ESMO), the San Antonio Breast Cancer Symposium (SABCS), and the St. Gallen Oncology Conferences, as well as relevant national meetings such as those of the Working Group on Gynecological Oncology (Arbeitsgemeinschaft Gynäkologische Onkologie, AGO).

\section{New Trends in Digital Tools for Knowledge Transfer}

Analyses of the usage of onkowissen.de (on Google Analytics) show that apps are evidently used in different ways from classic websites. Whereas the latter tend to be accessed in the evening and at weekends, apps on mobile devices are much more often used during consulting hours and visiting times. The mean time that users spend on individual articles is slightly shorter, but the numbers of return visits are much higher. Topical events such as the approval of a new drug or publication of a risk-benefit analysis have noticeable effects on the usage of the apps, since at the times when these happen they lead to more searches for rapidly accessible information about the agents concerned. These measurable trends, along with continuing increases in numbers of downloads, indicate that there is a clear need for digital knowledge transfer.

\section{Teaching and Council on Medical Education Tools}

Apps for oncological knowledge transfer will only persist in the longer term if they are kept constantly up to date and also incorporate additional requirements such as certification. In addition to the tools for treatment management mentioned above, the apps from onkowissen.de will also include sections on "knowledge/theory and training" that provide more in-depth information. In addition, teaching and Council on Medical Education tools are currently being developed that will enable users to familiarize themselves with a topic and take a digital examination. Applications for certification have been submitted to various state medical boards. The aim is to be able to offer a Council on Medical Education examina- 
tion as part of each app, which will be officially recognized by entering a Standard Further Education Number (Einheitliche Fortbildungsnummer, EFN).

\section{Are We Ready for Oncology 4.0?}

Digitalization obviously offers tremendous opportunities. However, risks and side effects also need to be taken into account - involving juridical, legal, ethical, and economic aspects, as well as psychological ones. What will the effects on health care be of telemedicine, wireless sensors, algorithms, and digital compliance control? What are the data protection issues involved? What effects on society are likely to occur? Are we ready yet for oncology 4.0? Are we able to deal with the resulting new opportunities in sufficiently responsible ways?

However, the central question will be: will the high standards that oncology sets for the personal physicianpatient relationship be endangered by digital tools, or will they perhaps even be optimized?

\section{Disclosure Statement}

F.O. is Managing Partner of onkowissen.de GmbH und Managing Partner of OncoConsult.Hamburg GmbH. F.O. received honoraria for speaking activity and consulting from Amgen, AstraZeneca, Bayer, BMS, Boehringer-Ingelheim, Chugai, Celgene, Cellex, ClinSol, Eisai, Gilead, Hexal, Iomedico, Ipsen, Janssen-Cilag, Merck, MSD, Novartis, Novonordisc, Riemser, Roche, Servier, Shire, Tesaro, and Teva.

\section{References}

1 Basch EM, Deal AM, Dueck AC, Bennett AV, Atkinson TM, Scher HI, et al. Overall survival results of a randomized trial assessing patientreported outcomes for symptom monitoring during routine cancer treatment. Paper presented at: American Society of Clinical Oncology 53rd Annual Meeting, Chicago, IL, June 2-6, 2017. J Clin Oncol. 2017;35:abstr LBA2.

2 Neckermann K, Schinköthe T. Adhärenz: Fehlende Therapietreue und Möglichkeiten der Steigerung. Forum. 2018;33(2):140-2.

3 Welslau M, Riese C, Beylich A, et al. Patients' competence in oral cancer therapies. Poster presented at: American Society of Clinical Oncology 52nd Annual Meeting, Chicago, IL, June 3-7, 2016. J Clin Oncol. 2016;34:abstr 6517.
4 Riese C. Patients' guidance with OncoCoaching and CANKADO on systemic chemotherapy for metastatic colorectal cancer (POSCA Study). Poster presented at: German Cancer Congress, Berlin, February 21-24, 2018. Deutscher Krebskongress 2018:abstr 562.

5 Matthies L, Taran F-A, Keilmann L, Schneeweiss A, Simoes E, Hartkopf AD, et al. Reliability of an ePRO Tool of FACT-B for Measuring Health-Related Quality of Life in Breast Cancer Patients. J Med Internet Res. Forthcoming 2018.

6 Wallwiener M, Matthies L, Simoes E, Keilmann L, Hartkopf AD, Sokolov AN, et al. Reliability of an e-PRO Tool of EORTC QLQC30 for Measurement of Health-Related Quality of Life in Patients With Breast Cancer: Prospective Randomized Trial. J Med Internet Res. 2017 Sep;19(9):e322.
7 Wallwiener M, Heindl F, Brucker SY, Taran FA, Hartkopf A, Overkamp F, et al. Implementation and Feasibility of Electronic Patient-Reported Outcome (ePRO) Data Entry in the PRAEGNANT Real-Time Advanced and Metastatic Breast Cancer Registry. Geburtshilfe Frauenheilkd. 2017 Aug;77(8):87078 .

8 Hartkopf AD, Graf J, Simoes E, Keilmann L, Sickenberger N, Gass P, et al. ElectronicBased Patient-Reported Outcomes: Willingness, Needs, and Barriers in Adjuvant and Metastatic Breast Cancer Patients. JMIR Cancer. 2017 Aug 7;3(2):e11. 